- Note-

\title{
Poisoning of HER Cathode Characterised by Isotopic Distribution and Current Density on Palladium Membrane Electrode
}

\author{
Hideaki YoshITAKE*, Go MUTO, Nobuyuki KAMIYA and Ken-ichiro OTA
}

Received June 2, 1997 ; Accepted August 26, 1997

\section{INTRODUCTION}

The surfaces of electrolysing electrodes often suffer poisonings which result from ions or molecules in the electrolytes. To estimate these poisoning is a difficult task because of the generation of bubbles which stick on the surface and the large electrolysing current which dominates over signals from the surface processes. These factors are obstructive to the application of the spectroscopies and the electrochemical analyses, respectively. Current-potential $(i-E)$ characteristics have been exclusively used for investigating the poisoning of the electrolyses in-situ. They are usually measured when the electrolysis reaches the "steady-state", which is rather conceptualised state for electrode surfaces in working. It is, however, often difficult to apply this concept since the reduction or oxidation of the surface is much more serious than those in the potential region for cyclic voltammetry. For example, we found $\mathrm{Li}^{+}$inclusion into $\mathrm{Pd}$ cathode during hydrogen evolution reaction (HER) in $\mathrm{LiOH}$ solution at relatively small polarization ${ }^{1)}$. This reaction proceeds as slow as metal atom diffusion in the other bulk metal at room temperature probably until the thermodynamically stable composition is achieved. In this case, it is almost impossible to find a "steady-state" of Pd surface during HER. For this reason, it would be important to investigate how the poisoning proceeds,

*Institute of Environmental Science \& Technology and Department of Energy Engineering, Yokohama National University, Hodogaya-ku, Yokohama 240, Japan.

Keywords: palladium, hydrogen evolution reactions, poisoning rather than the extent of it, which can be different among the elementary steps. We have developed a method utilising hydrogen isotopes on palladium membrane cathodes in HER ${ }^{2)}$. This requires a cell specially designed for offering a D-covered Pd surface by $\mathrm{D}_{2} \mathrm{O}$ electrolysis at the back face. The electrolyses of $\mathrm{H}_{2} \mathrm{O}$ by the front face produce hydrogen isotopes reflecting the elementary processes on the surface. Several isotopic parameters have been utilised for evaluating inhibitions of these processes. In this note we report the time dependence of these isotopic parameters as well as the current density of various electrolytes to characterise the progress of inhibitions.

\section{METHOD}

The construction of the cell for the electrolyses is illustrated in detail in ref. 2. A galvanostatic electrolysis $\left(1 \times 10^{4} \mathrm{~A} / \mathrm{m}^{2}\right.$ ) of $1 \mathrm{M} \mathrm{D}_{2} \mathrm{SO}_{4}$ started at the back face of Pd foil (thickness of $2.3 \times 10^{-5} \mathrm{~m}$ ) $30 \mathrm{~min}$ before the potentiostatic electrolysis of light water solutions at the front face starts (at $-0.4 \mathrm{~V}$ ). The electrolytes at the front face included sulphuric acid and that with $\mathrm{Li}^{+}$, tetrabutyl ammonium hydroxide (TBA), or $\mathrm{Zn}^{2+}$ as additives. The reference electrode was a reversible hydrogen electrode (RHE). The hydrogens formed at the front face were analysed by a quadrupole mass spectrometer. The delay of the detection from the gas formation was around $6 \mathrm{~min}$.

Isotopic distributions of $\mathrm{H}_{2}, \mathrm{HD}$ and $\mathrm{D}_{2}$ were transformed into three parameters. The current efficiency of $\mathrm{H}$ production which is a ratio of $\mathrm{H}$ formation rate (represented as current, $j_{H}$ ) to the current of the potentiostatic electrolysis, $j_{H} j_{f}$, the deuterium emission 
(represented as current, $j_{D}$ ) normalised by those for "poisoning-free surface," $j_{D} / j_{D}{ }^{0}$, and the extent of equilibration which is the ratio of measured molar fraction of $\mathrm{HD}(=x(\mathrm{HD}))$ to that expected for the complete shuffle $\left(x(\mathrm{HD})_{\mathrm{eq}}=2 x(\mathrm{H}) x(\mathrm{D})\right)^{3,4}$. These are functions of potential and time just like current density, $j_{f}$ It is to be noted that $j_{D}{ }^{0}$ cannot be given a priori. We thought that $j_{D}$ for the least poisoned surface is proper for $j_{D}{ }^{0}$. In this study, the value was defined as that at the open circuit potential in sulphuric acid.

\section{RESULTS AND DISCUSSION}

Figures from 1 to 4 show the time evolutions of the isotopic parameters and the current densities of the potentiostatic electrolyses. In the case of $\mathrm{H}_{2} \mathrm{SO}_{4}$ in figure 1 , except the first $20 \mathrm{~min}$, the suppression of $j_{D} / j_{D}{ }^{\circ}$ and $x(\mathrm{HD}) / x(\mathrm{HD})_{\text {eq }}$ were not observable, which showed that the emergence of deuterium from Pd bulk was not inhibited and the isotopes at the surface were shuffled completely. $j_{H} / j_{f}>1$ is explained by the reaction, $\mathrm{H}^{+}+$ $\mathrm{D}_{\text {ads }} \rightarrow \mathrm{H}_{\mathrm{ads}}+\mathrm{D}^{+}$(1). Three parameters $j_{H} / j_{f} j_{D} / j_{D}{ }^{0}$ and $j_{f}$ are coupled each other while $x(\mathrm{HD}) / x(\mathrm{HD})_{\text {eq }}$ became stable at $20 \mathrm{~min}$. In other words, before the surface state reaches the "steady state," the shuffle of the isotopes became perfect.

$$
x(\mathrm{HD}) / x(\mathrm{HD})_{\text {eq }} \text { was again almost constant at } 0.85 \text { after }
$$

$20 \mathrm{~min}$ in $\mathrm{Li}^{+}$added solution in Figure 2. On the contrary, $j_{D} / j_{D}{ }^{0}$ monotonously decreased, from 1.04 to 0.53 . This decrease seems coupled with $\mathrm{j}_{\mathrm{f}}$ which decreased from 820 $\mathrm{A} / \mathrm{m}^{2}(\mathrm{t}=0 \mathrm{~min})$ to $560 \mathrm{~A} / \mathrm{m}^{2}(\mathrm{t}=120 \mathrm{~min})$. The change did not agree with that of $j_{H} / j_{f}$, which decreased little, from 1.1 to 1.0 , i.e., adsorbed $\mathrm{H}$ is produced electrochemically and transformed into gaseous hydrogens. The mode of the poisoning is thus attributed to the inhibition of the adsorption of $\mathrm{H}$ and the emergence of $\mathrm{D}$ from the Pd bulk. This proceeds gradually, which is concordant with the slow inclusion process found by SIMS depth profile after the electrolysis ${ }^{1)}$. (a)
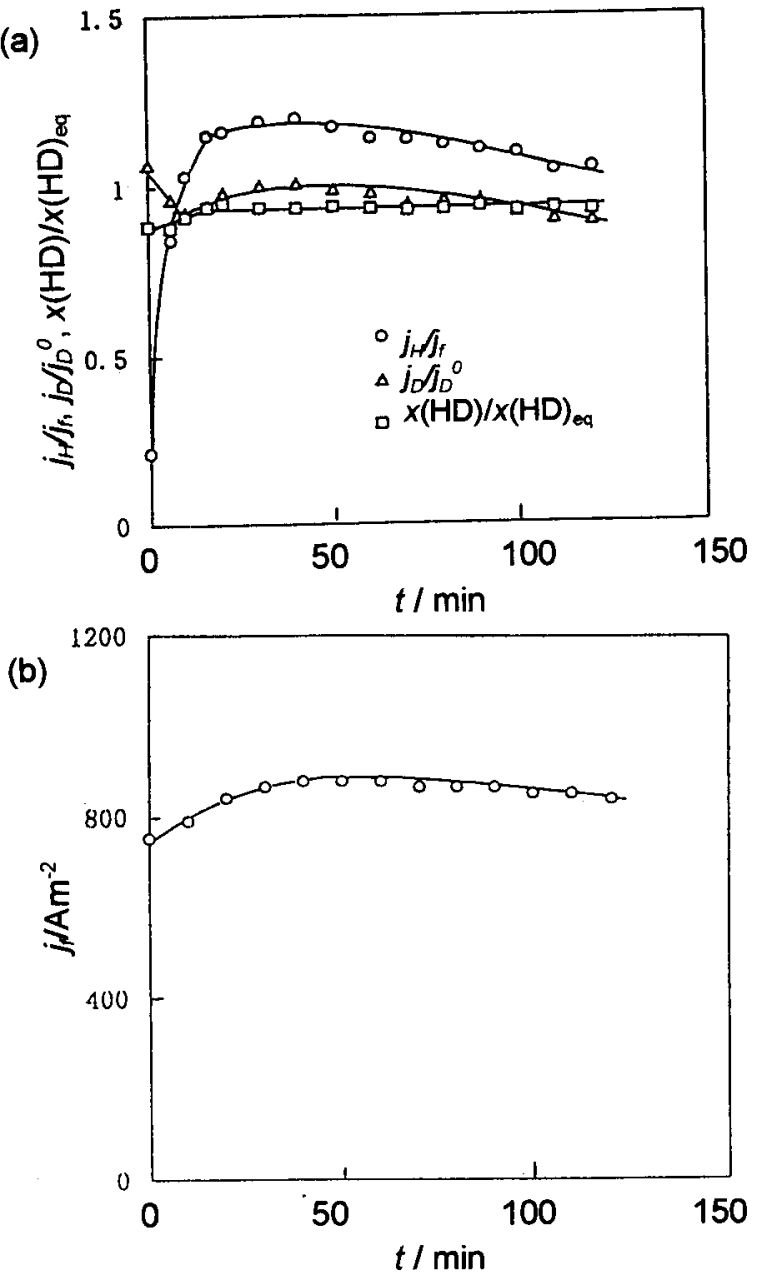

Fig. 1. Inhibition characteristics of HER in $0.5 \mathrm{M} \mathrm{H}_{2} \mathrm{SO}_{4}$. (a)Current efficiency of $\mathrm{H}$ formation, $j_{H} / j_{s}$ inhibition of deuterium emission, $j_{D} / j_{D}{ }^{0}$ and the extent of equilibration, $x(\mathrm{HD}) / x(\mathrm{HD})_{\mathrm{eq}}$. (b) Current density.

When the additive was TBA (Figure 3), $x(\mathrm{HD}) / x(\mathrm{HD})_{\mathrm{eq}}$ reached nearly unity at $20 \mathrm{~min}$ and kept constant. $j_{\mathrm{f}}$ increased monotonously, suggesting increase of $\mathrm{H}^{+}+e \rightarrow$ $\mathrm{H}_{\mathrm{ads}} . \quad j_{H} / j_{f}$ and $j_{D} / j_{D}{ }^{0}$ decreased once then increased whose local minimum appeared around $40 \mathrm{~min}$. These time dependence suggest the existence of the relatively slow reactions which reduce the poisoning of $\mathrm{H}$ absorption into Pd and D emergence from Pd. TBA is probably transformed on the surface from the early stage of the electrolysis and the products inhibit $\mathrm{H}$ formation, $\mathrm{H}$ 
absorption and $\mathrm{D}$ emission, but the activity as inhibitor is weaker than TBA itself. This possible transformation of the poison has no influence on the isotope mixing as proved in $x(\mathrm{HD}) / x(\mathrm{HD})_{\text {eq }} \fallingdotseq 1$.
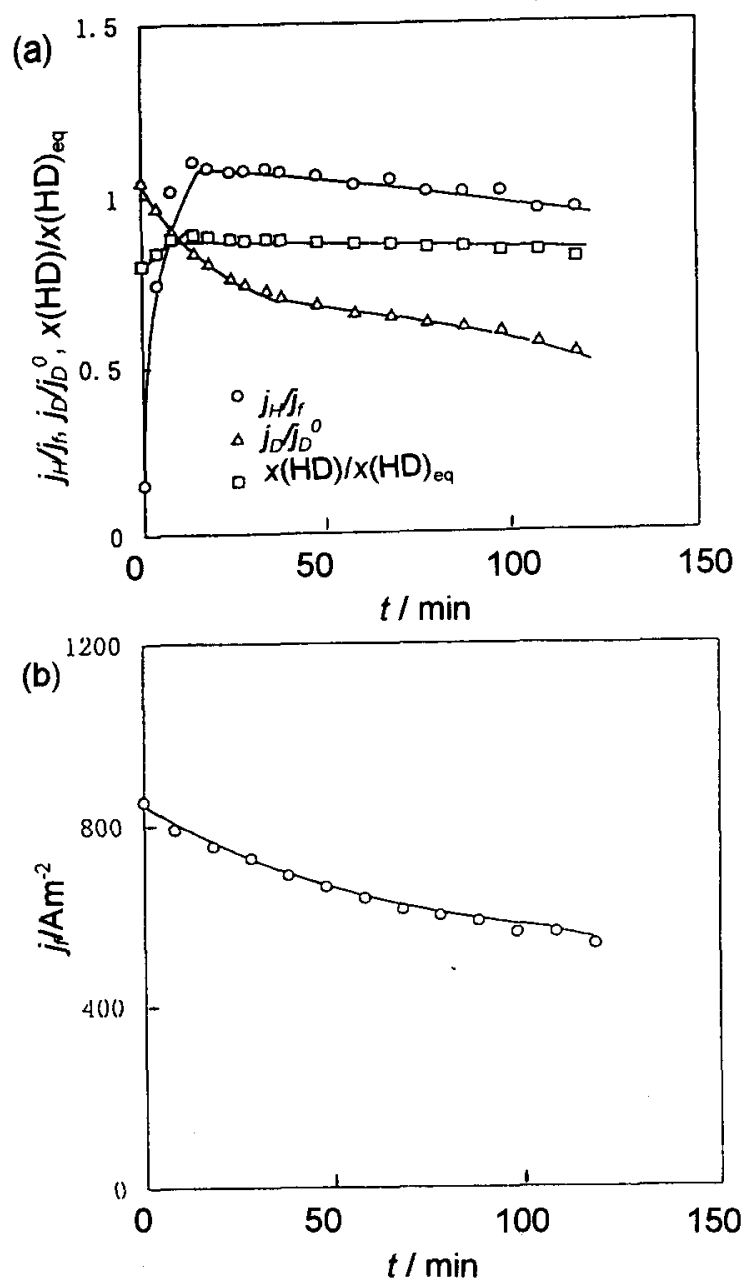

Fig. 2. Inhibition characteristics of $\mathrm{HER}$ in $0.5 \mathrm{M} \mathrm{H}_{2} \mathrm{SO}_{4}$ with $0.5 \mathrm{M} \mathrm{Li}^{+}$. (a)Current efficiency of $\mathrm{H}$ formation, $j_{H} / j_{s}$, inhibition of deuterium emission, $j_{D} / j_{D}{ }^{0}$ and the extent of equilibration, $x(\mathrm{HD}) / x(\mathrm{HD})_{\mathrm{eq}}$. (b) Current density.

The results of $\mathrm{Zn}$ added solution, Figure 4 , the shuffle parameter, $x(\mathrm{HD}) / x(\mathrm{HD})_{\text {eq, }}$ became stable around 0.9 within $20 \mathrm{~min}$, just as the other solutions. Rapid increases of $j_{H} / j_{f}$ and $j_{D} / j_{D}{ }^{0}$ were observed at the early stage of the electrolysis but they decreased after $20 \mathrm{~min}$ and reached around 1 at $120 \mathrm{~min}$. These complex changes cannot be attributed to the $\mathrm{Zn}$ accumulation because it occurs less than $-0.7 \mathrm{~V}$. The origin of the excess $\mathrm{H}$ formation until $120 \mathrm{~min}$ and the enhancement of $\mathrm{D}$ emission is difficult to be explained but the effect of $\mathrm{Zn}$ should be taken into account. The rate of the increase of $j_{f}$ and that of the decrease of $j_{H} / j_{f}$ are almost the same, showing constant production of $\mathrm{H}$. Non-electrochemical process of (1) and $\mathrm{H}$ absorption into bulk Pd may incidentally agree but it is not possible to confirm this assumption
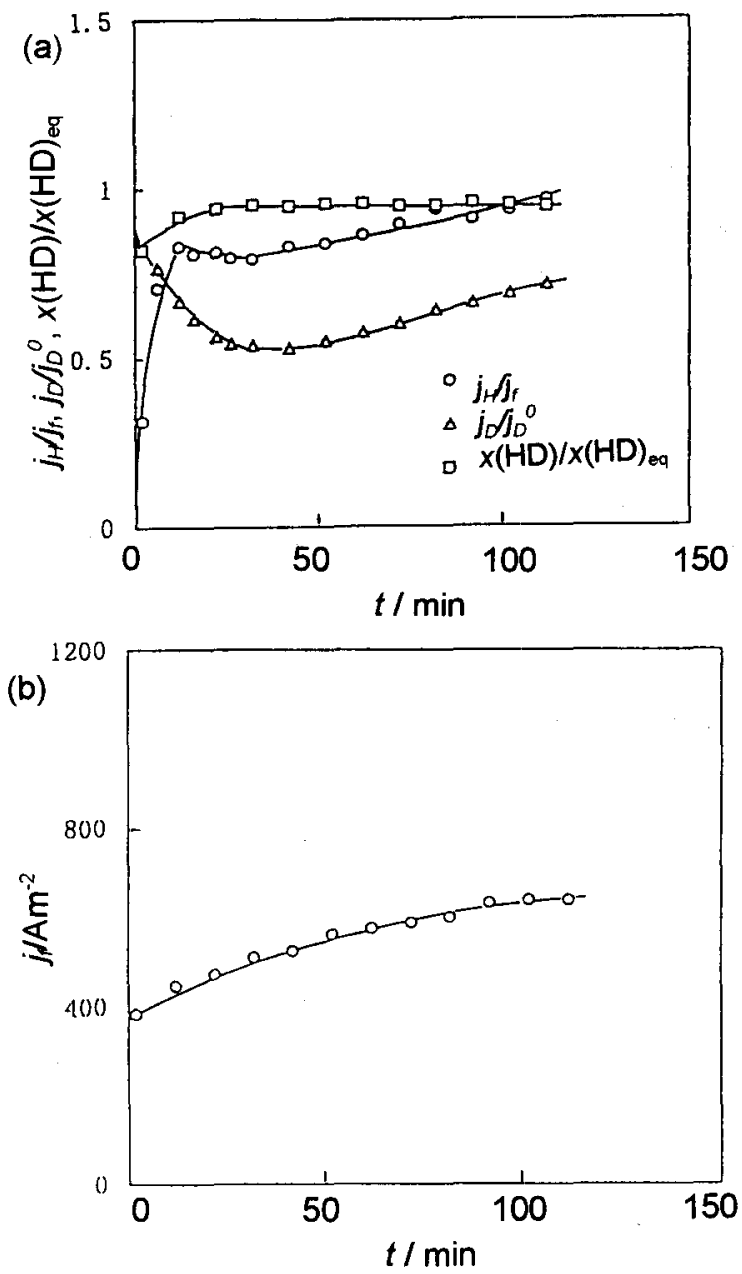

Fig. 3. Inhibition characteristics of HER in $0.5 \mathrm{M} \mathrm{H}_{2} \mathrm{SO}_{4}$ with $0.5 \mathrm{M} 1 \times 10^{-4} \mathrm{M}$ TBA. (a)Current efficiency of $\mathrm{H}$ formation, $j_{H} / j_{f}$ inhibition of deuterium emission, $j_{D} / j_{D}{ }^{0}$ and the extent of equilibration, $x(\mathrm{HD}) / x(\mathrm{HD})_{\mathrm{eq}}$. Current density. 

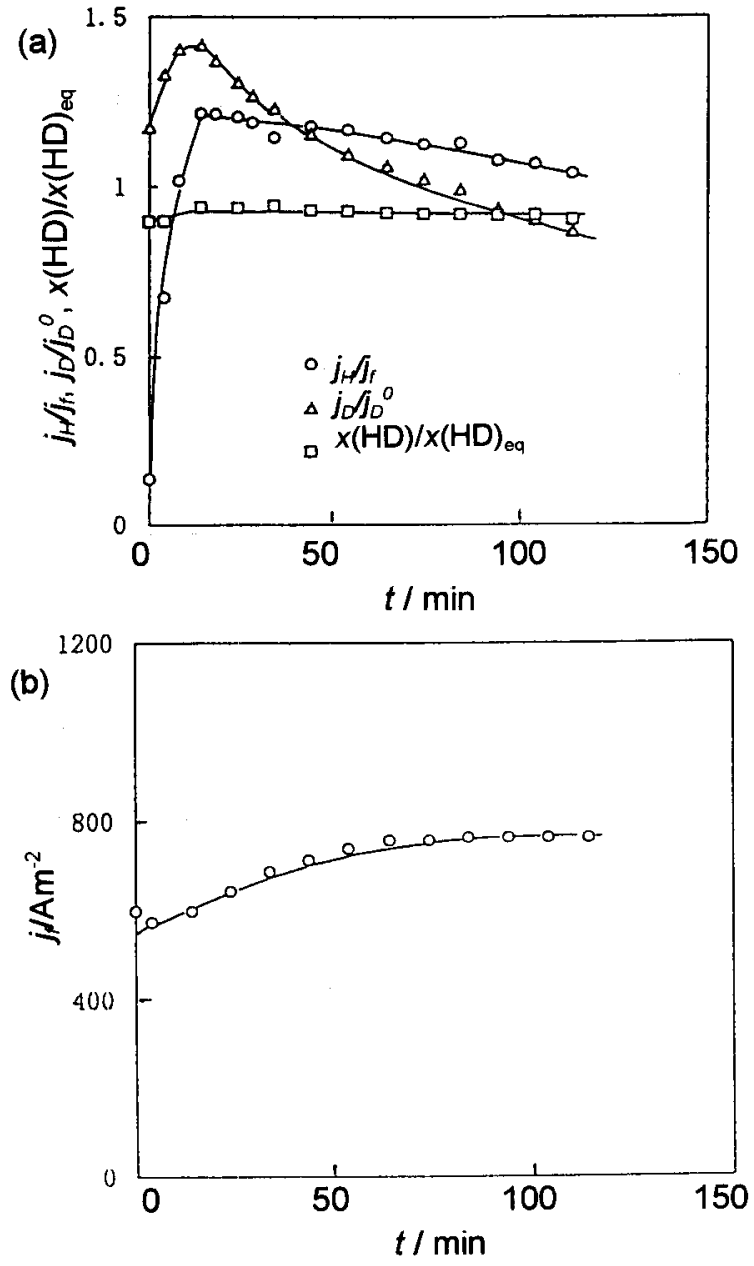

Fig. 4. Inhibition characteristics of HER in $0.5 \mathrm{M} \mathrm{H}_{2} \mathrm{SO}_{4}$

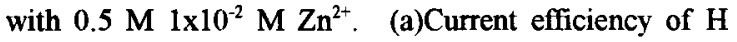
formation, $j_{H} j_{j}$, inhibition of deuterium emission, $j_{D} / j_{D}{ }^{0}$ and the extent of equilibration, $x(\mathrm{HD}) / x(\mathrm{HD})_{\mathrm{eq}}$. Current density.

because the high current density and the large production of $D_{2}$ prevents from gas analysis at the back face.

For all solutions, the current density, $j_{f}$ is a decreasing or increasing functions as are usually observed in the $i-E$ experiments. This can be a good indicator of the energy consumption of an electrolysis but the mechanism and the origin of the inhibition are ambiguous. $j_{H} / j_{f}$ and $j_{D} / j_{D}{ }^{0}$ can rapidly change before $20 \mathrm{~min}$ in general but this phenomenon is not observed in $x(\mathrm{HD}) / x(\mathrm{HD})_{\text {eq. }}$. This is due to the induction of the steady flow of the gas in the tube between the cell and the mass analyser. (The first two parameters are subject to the intensity of the mass signal but the last one is not.) Even except this induction period of the analyses, $j_{H} / j_{f}$ and $j_{D} / j_{D}{ }^{0}$ showed complex time evolution in TBA added solution whose origin is considered to surface transformations of TBA. This showed that these parameters can be more sensitive than the current density in the evaluation of poisonings. On the contrary, $x(\mathrm{HD}) / x(\mathrm{HD})_{\mathrm{eq}}$ easily became stable for all four solutions. The interpretation of this parameter is simpler than the other parameters; extent of diffusion and shuffling before desorption. As an indicator of poisonings, therefore, this is more easily usable than any others. The evaluation of poisoning by these parameters should be complementarily used according to the system and the aim of the experiment.

\section{REFERENCES}

1) O. Yamazaki, H. Yoshitake, N, Kamiya and K. Ota, $J$. Electroanal. Chem., 390, 127 (1995).

2) H. Yoshitake, G. Muto and K. Ota, J. Electroanal. Chem., 401, 81 (1996).

3) M. Enyo, Comprehensive Treatise of Electrochemistry, ( Eds. B. E. Conway, J. O'M Bockris, E. Yeager, S. U. M. Khan and R. E. White) vol. 7, Chapter 5, Plenum, New York (1983).

4) H. Yoshitake, T. Kikkawa and K. Ota, J. Electroanal. Chem, 390, 91 (1995). 